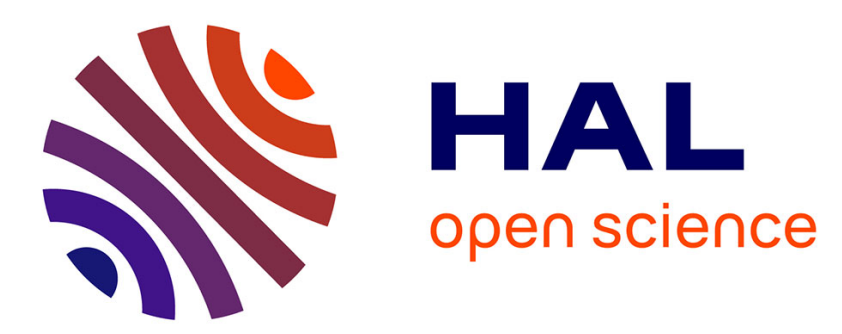

\title{
Evaluation of breast cancer service screening programme with a Bayesian approach: mortality analysis in a Finnish region
}

Jenny Chia-Yun Wu, Ahti Anttila, Amy Ming-Fang Yen, Matti Hakama, Irma Saarenmaa, Tytti Sarkeala, Nea Malila, Anssi Auvinen, Sherry Yueh-Hsia Chiu, Tony Hsiu-Hsi Chen

\section{To cite this version:}

Jenny Chia-Yun Wu, Ahti Anttila, Amy Ming-Fang Yen, Matti Hakama, Irma Saarenmaa, et al.. Evaluation of breast cancer service screening programme with a Bayesian approach: mortality analysis in a Finnish region. Breast Cancer Research and Treatment, 2009, 121 (3), pp.671-678. 10.1007/s10549009-0604-x . hal-00612977

\section{HAL Id: hal-00612977 https://hal.science/hal-00612977}

Submitted on 2 Aug 2011

HAL is a multi-disciplinary open access archive for the deposit and dissemination of scientific research documents, whether they are published or not. The documents may come from teaching and research institutions in France or abroad, or from public or private research centers.
L'archive ouverte pluridisciplinaire HAL, est destinée au dépôt et à la diffusion de documents scientifiques de niveau recherche, publiés ou non, émanant des établissements d'enseignement et de recherche français ou étrangers, des laboratoires publics ou privés. 


\title{
Evaluation of breast cancer service screening programme with a Bayesian approach: mortality analysis in a Finnish region
}

\author{
Jenny Chia-Yun Wu $\cdot$ Ahti Anttila $\cdot$ Amy Ming-Fang Yen • \\ Matti Hakama - Irma Saarenmaa - Tytti Sarkeala - Nea Malila • \\ Anssi Auvinen · Sherry Yueh-Hsia Chiu • Tony Hsiu-Hsi Chen
}

Received: 9 September 2009/ Accepted: 14 October 2009/Published online: 5 November 2009

(C) Springer Science+Business Media, LLC. 2009

\begin{abstract}
Evaluation of long-term effectiveness of population-based breast cancer service screening program in a small geographic area may suffer from self-selection bias and small samples. Under a prospective cohort design with exposed and non-exposed groups classified by whether women attended the screen upon invitation, we proposed a Bayesian acyclic graphic model for correcting self-selection bias with or without incorporation of prior information derived from previous studies with an identical screening program in Sweden by chronological order and applied it to an organized breast cancer service screening program in Pirkanmaa center of Finland. The relative mortality rate of breast cancer was 0.27 (95\% CI 0.12-0.61) for the exposed group versus the non-exposed group without adjusting for self-selection bias. With adjustment for selection-bias, the adjusted relative mortality rate without using previous data was 0.76 (95\% CI $0.49-1.15)$, whereas a statistically
\end{abstract}

J. C.-Y. Wu · M. Hakama - T. Sarkeala · N. Malila .

A. Auvinen - S. Y.-H. Chiu - T. H.-H. Chen

Tampere School of Public Health, University of Tampere,

Tampere, Finland

J. C.-Y. Wu

e-mail: chiayun.wu@gmail.com

M. Hakama

e-mail: matti.hakama@cancer.fi

T. Sarkeala

e-mail: Tytti.Sarkeala@uta.fi

N. Malila

e-mail: Nea.Malila@cancer.fi

A. Auvinen

e-mail: anssi.auvinen@uta.fi

S. Y.-H. Chiu

e-mail: sherrychiu@mail.cgu.edu.tw significant result was achieved [0.73 (95\% CI 0.57-0.93)] with incorporation of previous information. With the incorporation of external data sources from Sweden in chronological order, adjusted relative mortality rate was $0.67(0.55-0.80)$. We demonstrated how to apply a Bayesian acyclic graphic model with self-selection bias adjustment to evaluating an organized but non-randomized breast cancer screening program in a small geographic area with a significant $27 \%$ mortality reduction that is consistent with the previous result but more precise. Around $33 \%$ mortality was estimated by taking previous randomized controlled data from Sweden.

Keywords Breast cancer screening - Self-selection bias . Bayesian acyclic graphic model $\cdot$ Mortality reduction

A. Anttila $\cdot$ M. Hakama $\cdot$ N. Malila

Mass Screening Registry, Finnish Cancer Registry, Helsinki,

Finland

A. Anttila

e-mail: Ahti.Anttila@cancer.fi

I. Saarenmaa

Pirkanmaa Cancer Society, Tampere, Finland

e-mail: irma.saarenmaa@fimnet.fi

A. M.-F. Yen · T. H.-H. Chen ( $₫)$

Division of Biostatistics, College of Public Health,

National Taiwan University, Room 533, No. 17,

Hsu-Chow Road, Taipei 100, Taiwan

e-mail: chenlin@ntu.edu.tw

A. M.-F. Yen

e-mail: amyyen@ntu.edu.tw 


\section{Introduction}

The Finnish nationwide breast cancer service screening programme for women aged 50-59 years every 2 years has been started since 1987 and successfully implemented until now. Good quality of this service screening programme has been reported $[1,2]$ and the early evaluation of the effectiveness of the breast cancer screening regime during the first 3 years of this service screening program by comparing the invited women with the control cohort who have not been invited to screen yet showed a $24 \%$ non-significant mortality reduction for the invited women [3]. Since the effectiveness of breast cancer screening is subject to many factors (such as age, attendance rate, and inter-screening interval), it therefore suggests that any population-based organized service screening even small areas should be evaluated once launched. However, it is very difficult to evaluate long-term effectiveness of breast cancer service screening program because no concurrent controls are available. In addition, evaluation of long-term effectiveness in small areas may also suffer from insufficient statistical power.

Here, we evaluate long-term effectiveness by comparing cumulative mortality from breast cancer between attender and non-attender in such a small population covered by an organized screening programme. We adjust the results for the self-selection in attendance and incorporate extraneous information by a Bayesian acyclic model to reduce bias and increase precision. Our data stems from 2-yearly breast cancer screening program implemented between 1988 and 2000, in Pirkanmaa center, Finland.

\section{Materials and methods}

\section{Study subjects}

Ten screening centers of the Cancer Society of Finland have been responsible for breast cancer screening data which covered half of the target population in Finland [1, 4]. The data used in this study are derived from one of these screening centers, the Cancer Society of Pirkanmaa center in Tampere, Finland. The data covers invitations between 1988 and 2000.

\section{Data}

To elucidate the effect of biennial screening regime on breast cancer mortality, we used a prospective cohort design, where the cohort invited to screening is divided into two groups: the exposed and the non-exposed groups depending on whether women attended (exposed) to screen. Women who were invited but never came to screen were classified as the non-exposed group. After the average of five invitations, the number of non-exposed group accounted for about $6.5 \%$ among the invited women.

In this study, screen-detected breast cancer and interval cancer, the cancer diagnosed between screening rounds, were classified as diagnosed in the exposed group. The interval cancer included also the women who had been diagnosed with breast cancer any time after a negative screening as women may not regularly attend again after attending a given round of screen. Interval cancers and cases diagnosed in the non-exposed group were identified through the linkage to the Finnish cancer registry. Only deaths from breast cancer diagnosed after first recorded invitation for non-exposed or screen (exposed) were included in the analysis (deaths of breast cancer diagnosed prior to start of follow-up were excluded), which implies the refined mortality (and only incident tumors diagnosed in the study period were included) was the outcome of interest. The inclusion of only incident tumors could avoid the contamination of the screening-exposure time by the screening-nonexposure time to see the real effect of screening.

Mortality and incidence analysis

The calculation of person-years of mortality for the exposed and the non-exposed groups was done using individual record from the date of first invitation for nonexposed and first actual screen for exposed until the date of death from breast cancer, or the date of death due to other competing causes, migration, or the end of study in the year 2000, whichever came first. The refined mortality of breast cancer during the study period was calculated separately for the exposed and the non-exposed groups. The curve of the cumulative mortality of breast cancer between the two groups was plotted for visual inspection. Poisson regression model was used to calculate relative mortality rate of breast cancer for the exposed against the non-exposed group and its $95 \%$ CI. Note that the calculation of person-years for incidence rate was still based on individual record from the date of first invitation for non-exposed and first actual screen for exposed until the date of diagnosis as breast cancer, the date of death due to breast cancer or other competing causes, migration, the age beyond 59 years (the upper limit of age of screening in Finland), or the end of study in the year 2000 , whichever came first.

Bayesian model for self-selection adjustment

We estimated the extent of mortality reduction from breast cancer as a result of screening regime with adjustment for selection bias using Bayesian acyclic graphic model underpinning the framework of generalized linear model whereby Poisson regression model was constructed. The details are explained in detail in the Appendix. As 
illustrated in Eq. 1 (Appendix), the baseline group for selfselection bias adjustment is the uninvited group with the background breast cancer mortality equivalent to 21.1/ 100,000 (63 deaths among the 299,228 women-years) derived from Hakama et al. study [3]. The reason for using Bayesian approach is that as Pirkanmaa is one of local areas that have organized screening program within nationwide screening program since 1987, evaluation of long-term effectiveness in mortality reduction may be subject to insufficient sample size. To overcome this problem, analysis can be strengthened with the incorporation of information from the previously published early 3 years of nationwide screening program which is supposed to have a similar screening program that warrants the exchangeability of data between the Pirkanmaa data and the prior information. From a statistical viewpoint, this provides a justification for the use of Bayesian approach to enhance statistical power without mitigating the validity of results. Consequently, two types of prior, non-informative and informative priors are incorporated in the Bayesian acyclic graphic model. The former is simply based on the data from the current screening program in Pirkanmaa center, whereas the latter uses data from Pirkanmaa screening and the external information from nationwide study that reported a 0.76 relative rate of death from breast cancer in the birth cohorts invited opposed to the uninvited birth cohorts [3]. Second, we considered how to apply the Bayesian approach to sequentially incorporate prior information from previous randomized studies in Sweden. The details are given in Appendix. Adjusted relative rates of reduction in breast cancer mortality and the corresponding
95\% CI were implemented using Markov Chain Monte Carlo (MCMC) method with Gibbs sampling technique.

\section{Results}

A total of 110,299 invitations and 98,049 screening visits among 36,697 women were identified and recorded individually during this period, including 95,057 invitations and 84,812 screening visits for women aged 50-59 years at the time of invitation with the corresponding attendance rate by calendar time from 1988 to 2000 , ranging between 86 and $90 \%$ (Table 1) commensurate with the previous report [5]. Note that such figures were not exactly the same as the proportion of women exposed to screen in the following incidence and mortality analysis that followed up total of 33,375 women, consisting of 31,192 exposed to screen and 2,183 non-exposed.

A total number of 513 invasive breast cancer patients were ascertained. Of 513 patients, 64 cancers were diagnosed before their first screening round and were excluded from the following analysis. Table 2 lists numbers of breast cancers and death by detection modes. The total of 130 first screen-detected invasive cancers and 159 subsequent screen-detected invasive cancers were identified through the organized mammography screening regime by excluding one carcinoma in situ (CIS) detected from first screen and four CIS detected from subsequent screens. A total of 140 interval cancers were identified. 20 breast cancer patients derived from the women who have never come to the organized screening program. Therefore, a

Table 1 Number of invitations, attendants, and the attendance rate in the Pirkanmaa center, Finland, 1988-2000

\begin{tabular}{lcccccccccccccc}
\hline & 1988 & 1989 & 1990 & 1991 & 1992 & 1993 & 1994 & 1995 & 1996 & 1997 & 1998 & 1999 & 2000 & Total \\
\hline Total & & & & & & & & & & & & & & \\
Invitations & 4553 & 5039 & 7142 & 6452 & 8024 & 6923 & 6885 & 8183 & 7558 & 7910 & 8125 & 9665 & 8598 & 95057 \\
Attendants & 4005 & 4358 & 6340 & 5728 & 7187 & 6162 & 6176 & 7288 & 6779 & 7018 & 7309 & 8677 & 7786 & 84812 \\
Attendance rate (\%) & 87.96 & 86.49 & 88.77 & 88.78 & 89.57 & 89.01 & 89.70 & 89.06 & 89.69 & 88.72 & 89.96 & 89.78 & 90.56 & 89.22 \\
\hline
\end{tabular}

Table 2 Number of invasive breast cancer and death of breast cancer (BC) by detection mode and age group in the Pirkanmaa center, Finland, 1988-2000

\begin{tabular}{|c|c|c|c|c|c|c|c|c|c|c|}
\hline \multirow[b]{3}{*}{ Age } & \multicolumn{8}{|l|}{ Exposed } & \multicolumn{2}{|c|}{ Non-exposed } \\
\hline & \multicolumn{2}{|c|}{ First screen-detected cases } & \multicolumn{2}{|c|}{ Subsequent screen-detected cases } & \multicolumn{2}{|c|}{ Interval cases ${ }^{\mathrm{a}}$} & \multicolumn{2}{|c|}{ Interval cases ${ }^{\mathrm{b}}$} & \multirow[b]{2}{*}{ BC death } & \multirow[b]{2}{*}{ No } \\
\hline & BC death & No. & BC death & No. & BC death & No. & BC death & No. & & \\
\hline $50-54$ & 5 & 101 & 4 & 65 & 6 & 65 & 0 & 2 & 1 & 9 \\
\hline $55-59$ & 0 & 29 & 2 & 94 & 11 & 64 & 1 & 9 & 6 & 11 \\
\hline Total & 5 & 130 & 6 & 159 & 17 & 129 & 1 & 11 & 7 & 20 \\
\hline
\end{tabular}

\footnotetext{
${ }^{\mathrm{a}}$ Time since last negative screening $\leq 2$ years

b Time since last negative screening $>2$ years
} 


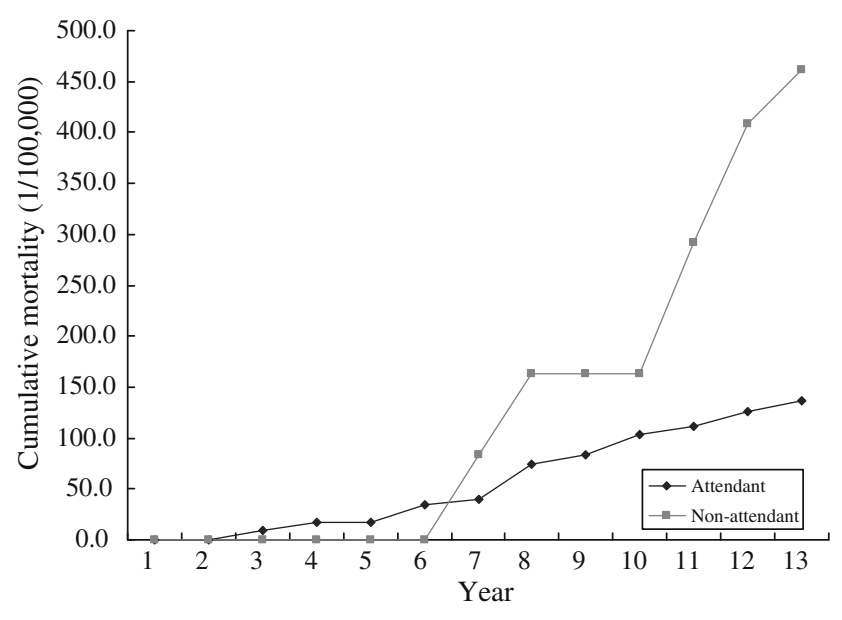

Fig. 1 Cumulative mortality of breast cancer death by calendar year in Pirkanmaa center, 1988-2000

total of 429 breast cancer patients were classified into the exposed group, 20 breast cancer patients were classified into non-exposed group.

Given 147,909 person-years observed in exposed group and 7,419 in the non-exposed group regarding the incidence, annual incidence rates of invasive breast cancer were estimated as 290/100,000 and 270/100,000 for the two groups, respectively. Given 233,991 person-years regarding the mortality in the exposed group and 14,966 in the non-exposed group, the estimation of mortality of breast cancer during the study period was $12.4 / 100,000$ person-years and 46.8/100,000 person-years for the exposed and non-exposed groups, respectively. The cumulative mortality of breast cancer is shown in Fig. 1, which shows higher mortality for the non-attendant group when compared to the attendant group has started since 1994, which is also approximately 6 years after the introduction of mass screening. The relative rate was 0.27 (95\% CI 0.12-0.61) for the comparison between the exposed and the non-exposed groups using a Poisson regression model.

Table 3 lists the details of prior, data used for likelihood, and posterior estimates using Bayesian approach. If information is only based on data from Finland, given data from Pirkanmaa data with non-informative prior for all regression coefficients (without referring to the previous result of Hakama study), adjusted RR (adjustment for selection bias) for the exposed group against the non-exposed group was 0.76 (95\% CI 0.49-1.15), suggesting that mammographic service screening in Pirkanmaa reduced 24\% the breast cancer mortality, which was not statistically significant. With informative prior regression coefficients derived from the early 3 years of organized screening service program from the nationwide study adjusted RR was 0.73 (0.570.93 ), suggesting a significant $27 \%$ reduction in breast cancer mortality.

Table 3 shows the estimated results using Bayesian sequential approach outside Finland. With the incorporation of successive information in chronological order, including Dalarna study [9], Gothenburg study [10] in Sweden, the early nationwide study in Finland [3], the adjusted RR based on these sequential priors together with the Pirkanmaa data was $0.67(0.55-0.80)$.

Table 3 The estimated results of relative mortality rate adjusted by self-selection bias with Bayesian acyclic graphic model

\begin{tabular}{|c|c|c|c|}
\hline External sources & $\begin{array}{l}\text { Types of prior and } \\
\text { prior distributions }\end{array}$ & $\begin{array}{l}\text { Data for likelihood/posterior } \\
\text { distributions* }\end{array}$ & $\begin{array}{l}\text { Adjusted } \\
\text { RR }(95 \% \mathrm{CI})\end{array}$ \\
\hline \multicolumn{4}{|l|}{ 1. Finland data only } \\
\hline & $\begin{array}{l}\text { Non-informative Prior-all regression } \\
\text { coefficients are assigned as } N\left(0,10^{6}\right)\end{array}$ & $\begin{array}{l}\text { Pirkanmaa/ } \alpha \sim\left(-8.47,0.13^{2}\right) \\
\quad \beta_{1} \sim\left(-0.54,0.23^{2}\right) \beta_{2} \sim\left(0.73,0.41^{2}\right)\end{array}$ & $0.76(0.49-1.15)$ \\
\hline & $\begin{array}{l}\text { Informative prior-from Finland } / \alpha \sim(-8.47 \text {, } \\
\left.0.13^{2}\right) \beta_{1} \sim\left(-0.41,0.19^{2}\right) \beta_{2} \sim\left(0.30,0.29^{2}\right)\end{array}$ & $\begin{array}{l}\text { Pirkanmaa/ } \alpha \sim\left(-8.47,0.11^{2}\right) \\
\quad \beta_{1} \sim\left(-0.48,0.14^{2}\right) \beta_{2} \sim\left(0.46,0.25^{2}\right)\end{array}$ & $0.73(0.57-0.93)$ \\
\hline \multicolumn{4}{|l|}{ 2. Outside Finland } \\
\hline $\begin{array}{l}\text { (1) Tabár et al./1977/ } \\
\text { Dalarna in Sweden }\end{array}$ & $\begin{array}{l}\text { Non-informative prior-all regression } \\
\text { coefficients are assigned as } N\left(0,10^{6}\right)\end{array}$ & $\begin{array}{l}\text { Dalarna/ } \alpha \sim\left(-7.79,0.16^{2}\right) \\
\quad \beta_{1} \sim\left(-0.92,0.25^{2}\right) \beta_{2} \sim\left(-0.05,0.40^{2}\right)\end{array}$ & $0.46(0.29-0.72)$ \\
\hline $\begin{array}{l}\text { (2) Bjurstam et al./ } \\
\text { 1982/Gothenburg in } \\
\text { Sweden }\end{array}$ & $\begin{array}{l}\text { Informative prior-from the posterior } \\
\text { distribution of }(1) / \alpha \sim\left(0,10^{6}\right) \beta_{1} \sim(-0.92, \\
\left.0.25^{2}\right) \beta_{2} \sim\left(-0.05,0.40^{2}\right)\end{array}$ & $\begin{array}{l}\text { Gothenburg/ } \alpha \sim\left(-6.99,0.11^{2}\right) \\
\quad \beta_{1} \sim\left(-0.64,0.18^{2}\right) \beta_{2} \sim\left(0.25,0.25^{2}\right)\end{array}$ & $0.69(0.51-0.92)$ \\
\hline $\begin{array}{l}\text { (3) Hakama et al./ } \\
\text { 1987/Finland }\end{array}$ & $\begin{array}{l}\text { Informative prior-from the posterior of }(2) / \\
\alpha \sim\left(0,10^{6}\right) \beta_{1} \sim\left(-0.64,0.18^{2}\right) \beta_{2} \sim(0.25 \\
\left.0.25^{2}\right)\end{array}$ & $\begin{array}{l}\text { Finland } / \alpha \sim\left(-8.42,0.10^{2}\right) \\
\quad \beta_{1} \sim\left(-0.53,0.13^{2}\right) \beta_{2} \sim\left(0.26,0.19^{2}\right)\end{array}$ & $0.70(0.56-0.86)$ \\
\hline $\begin{array}{l}\text { (4) Current study/ } \\
\text { 1988/Pirkanmma in } \\
\text { Finland }\end{array}$ & $\begin{array}{l}\text { Informative prior-from the posterior } \\
\text { distribution of }(3) / \\
\alpha \sim\left(-8.42,0.10^{2}\right) \beta_{1} \sim\left(-0.53,0.13^{2}\right) \\
\beta_{2} \sim\left(0.26,0.19^{2}\right)\end{array}$ & $\begin{array}{l}\text { Pirkanmma/ } \alpha \sim\left(-8.41,0.09^{2}\right) \\
\quad \beta_{1} \sim\left(-0.55,0.11^{2}\right) \beta_{2} \sim\left(0.34,0.17^{2}\right)\end{array}$ & $0.67(0.55-0.80)$ \\
\hline
\end{tabular}

* Normal distribution denoted by $N\left(\right.$ mean, standard deviation $\left.{ }^{2}\right)$ 


\section{Discussion}

After the era of evidence-based medicine on breast cancer screening between 1970 and mid-1990, organized breast cancer service screening programmes have been suggested as a national policy in several Western countries since then. There is an increasing demand for evaluation of organized breast cancer service screening program outside of the experimental environment at either national or local level in many other countries with relatively recently instituted screening programs. However, as the effectiveness of breast cancer screening is affected by a number of determinants it is therefore rather heterogeneous and varies from place to place. Bias arising from non-experimental study design such as self-selection bias, a major threat to evaluation of breast cancer service screening program, further complicates evaluation. In addition, evaluation of a wellorganized service screening program in a small local area also suffers from small sample size as well as the absence of a control group.

Using Bayesian approach and design without uninvited controls, this study demonstrates how to evaluate long-term effectiveness of an organized breast cancer service screening program. The method was applied to a subpopulation in Finland by exploiting prior information based on nationwide data. This approach is particularly valuable for evaluation of long-term effectiveness of organized breast cancer service screening in a small area. Selection bias can be estimated only when there are controls not covered by screening. Without considering the bias involved in selfselection in attendance, the estimate of effect was $73 \%$. After adjustment for selection-bias without referring to prior information on effect in mortality from early 3 years of nationwide program, the effectiveness of a biennial mammographic service screening (given $90 \%$ attendance rate) in the reduction of breast cancer mortality was $24 \%$ but non-significant. However, with the incorporation of prior information from early 3 years of nationwide program the benefit was similar (26\%) and become statistically significant.

\section{Credibility of correction for self-selection bias}

In contrast to previous studies on the evaluation of organized service screening based on the control group either from the uninvited group (group not covered by screening) [3] or prescreening period [6], the comparator group used in this study is based on the non-responders to the invitation. According to previous studies, the non-attenders in Finnish organized mammography program comprised two subgroups: one subgroup was more susceptible to breast cancer and found outside mammography screening elsewhere, the other subgroup was less compliant with health recommendations and services and was real non-attenders [7]. There are two major advantages to use non-attenders as the comparator although the women who were invited with less numbers of screen times may have higher likelihood of being categorized to the non-attenders and may be correlated with shorter follow-up time. There are no uninvited subjects as in Hakama et al. study [3] as the control group after full-blown screening program. Moreover, using contemporaneously diagnosed and treated breast cancer patients also ensures the effect observed was not attributed to the changes over time in therapy or increasing incidence with time due to biological property that are so-called history effect, one of threats to internal validity in quasi-experimental studies [8]. The use of pre-screening period like Parvinen et al. [6] study as the control group may be subject to this history effect. On the other hand, using non-attendants as the comparator are apt to self-selection bias. Bayesian approach for correcting this bias is one of major subjects in this study.

\section{Usefulness of Bayesian approach}

To the best our knowledge, this is the first time to consider using Bayesian graphic model for evaluation of organized breast cancer service screening program. Bayesian analysis is particularly useful for analyzing small-area data by taking other data sources as prior information from the population subjected to the same health services to increase precision without comprising the validity. In our study, we borrowed information [3] from the previous study covering all Finland to get the posterior distribution to make the adjusted relative risk more precise as compared with that without using prior information.

In addition to data related to the same service, this approach can also take into account other data sources outside the service. In such a case, however, the interpretation is different as the objective is to find an average, global estimate of effect rather than to improve the precision of the study with poor power. This sequential approach of Bayesian approach is to calculate posterior adjusted relative rate in the light of meta-analysis principle, taking into account the heterogeneity across studies with the incorporation of a random effect $\left(b_{i}\right)$ as illustrated in Eq. 1. The meta-analysis of three studies (Dalarna-county trial [9], Gothenburg trial [10], and Finnish nationwide program [3]) that showed a wide range of relative rates, $0.46,0.91$, and 0.76 , for three studies yielded 0.67 ( 0.55 0.80 ) of relative rate for the risk of breast cancer death and the random effect $\left(b_{i}\right)$ was statistically significant. It should be noted that one cannot use global meta-analysis for a specific population. Therefore, we do not recommend the use of results of meta-analysis only as the basis of decision to screen or not without the intention to estimate the effect also in the specific target population. 


\section{Model validation}

To validate whether the effectiveness after correcting for self-selection bias is closer to an unbiased estimate, we searched for the published literatures regarding the randomized controlled trial of breast cancer screening trial for age group 50-59 years from Nordic country that used the uninvited women as the equivalent control group as if they were derived from the randomized controlled trial. The randomized controlled trials included Dalarna-county trial [9], Gothenburg trial [10], and Finnish nationwide study [3] that screened first women born in even cohorts (newborn by allocated between them and odd-born cohort). If data are only based on the invited to screen arm, the relative rate after the correction of self-selection bias in Gothenburg trial for aged 50-59 years was $0.93(0.60,1.34)$, which was close to the corresponding figure, 0.91 (0.61-1.36), calculated by the comparison between the invited and the uninvited in light of intention-to-treat principle in a randomized controlled trial. The similar finding was found in the Dalarna-county trial. The corresponding figures were $0.47(0.29-0.72)$ and $0.46(0.29-0.73)$ for women aged 5059 years in Dalarna. In Finland nationwide program, the relative rate was estimated as 0.76 [3] which was close to our estimate using Bayesian approach 0.77 (0.54-1.10). This finding supports the elimination of self-selection bias using our Bayesian approach.

In conclusion, we proposed a Bayesian acyclic graphic model with self-selection bias adjustment to evaluate an organized breast cancer screening program in a subregion in Finland. The results indicated that with the expedient use of prior information we demonstrated a significant $27 \%$ mortality reduction that formally combines the observational small-area results with previous information on the service and is more precise than estimates without use of prior information. We also demonstrate how to sequentially use the acyclic Bayesian graphic model to have a global estimate of effect that corresponds to metaanalysis.

Acknowledgment This research study was supported by the FiDiPro Research Project of Tampere School of Public Health Granted from Academy of Finland

\section{Appendix: Bayesian acyclic graphic model for adjusting for selection-bias}

A Bayesian acyclic graphic model is proposed to estimate adjRR and its $95 \%$ CI. The detailed notations and method are elaborated as follows:

Let $Y_{i j}$ denote the observed numbers of breast cancer death in the $i$ th study with the $j$ detection mode $(j=1$ for exposed, 2 = non-exposed, and 3 = uninvited). Hence, $Y_{i j}$ follows a Poisson distribution with the expected value of $\mu_{i j}$. Attendance rate $\left(r_{\mathrm{i}}\right)$ in the $i$ th study is treated as a random variable specified by a beta distribution, Beta ( $\left.N_{i, \text { exposed }}, N_{i, \text { non-exposed }}\right) . N_{i, \text { exposed }}$ and $N_{i, \text { non-exposed }}$ are numbers of the exposed and the non-exposed groups, respectively, from the $i$ th study (see Fig. 2).

Following the framework of generalized linear model, the relationship between the outcome $Y$ and detection modes was regressed through a logarithm link function like the following:

$\log \left(\frac{\mu_{i, j}}{{\text { Person }- \text { years }_{i, j}}}\right)=\alpha+\beta_{1} \times I_{S}+\beta_{2} \times I_{\bar{S}}+b_{i}$
Fig. 2 A Bayesian acyclic graph model for self-selection bias adjustment

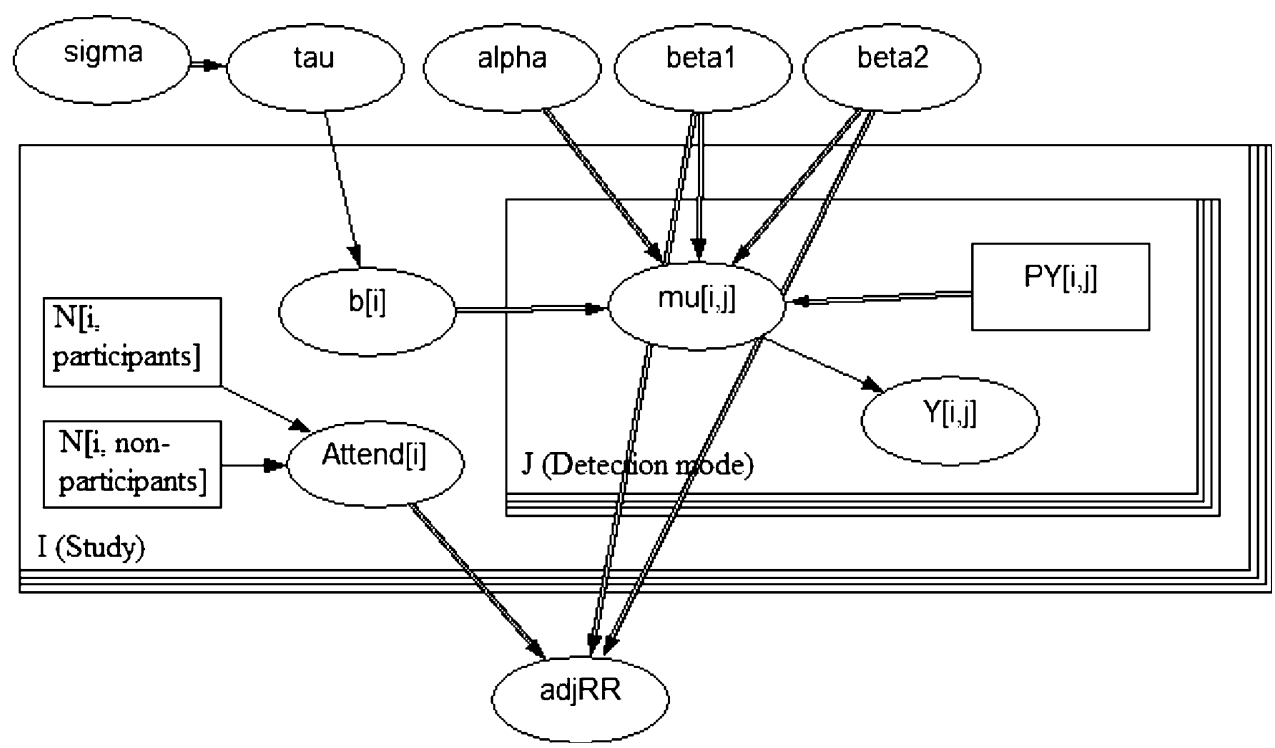


$I_{S}$ and $I_{\bar{S}}$ are two indicator variables for participant and non-participant, respectively, opposed to the uninvited group (baseline group). Two hyperparameters, $\beta_{1}$ and $\beta_{2}$, are the corresponding coefficients indicating the magnitudes of the risk for breast cancer death for the participant and the non-participant groups, respectively, compared with the uninvited group. Note that $b_{i}$ is a latent variable (random-effect) for capturing the heterogeneity across studies. We suppose $b_{i}$ follows a normal distribution with 0 and tau as the mean value and the inverse of variance $\left(\sigma^{2}\right)$.

Once $\beta_{1}$ and $\beta_{2}$ were estimated, we calculated the adjusted relative risk and its $95 \% \mathrm{CI}$ using the following formula:

$\operatorname{adjRR}=r \% \times \exp \left(\beta_{1}\right)+(1-r \%) \times \exp \left(\beta_{2}\right)$

Equation 2 is similar to previous formula used for adjusting self-selection bias [11, 12]: $\left(r \% \times P_{S}+(1-r \%) \times\right.$ $\left.P_{\bar{S}}\right) / P_{\bar{I}}$, which is a function of attendance rate $(r \%)$ and the mortality ratios for the participant group $\left(P_{s}\right)$ and the nonparticipant group $\left(P_{\bar{S}}\right)$ compared to the uninvited group $\left(P_{\bar{I}}\right)$. By comparing our Eq. 2 with the previous method, there are two equivalents: $\exp \left(\beta_{1}\right)=P_{S} / P_{\bar{I}}$ and $\exp \left(\beta_{2}\right)=$ $P_{\bar{S}} / P_{\bar{I}}$.

This Poisson regression model was constructed by an acyclic graphic model as shown in Figure 2. Following Spiegelhalter et al. [13], the oval nodes stand for stochastic nodes and square nodes for constant observed values. The single solid arrow captures the stochastic relationships from parents' nodes to their offsprings' ones and the double-lined arrow represents the logic link without stochastic property. With the acyclic graphic model, one can establish the relationships among observed data, random variables, and unobserved parameters by specifying the joint probability. In this case, the joint probability is expressed as:

$$
\begin{aligned}
& P\left(\mu_{i j}, \alpha, \beta_{1}, \beta_{2}, b_{i}, \tau, \operatorname{adjRR}, r_{i}\right) \propto \\
& \quad \prod_{i} \prod_{j} P\left(\mu_{i j} \mid \alpha, \beta_{1}, \beta_{2}\right) \times P\left(\operatorname{adjRR} \mid \alpha, \beta_{1}, \beta_{2}, r_{i}\right) \\
& \quad \times P(\alpha) \times P\left(\beta_{1}\right) \times P\left(\beta_{2}\right) \times P\left(r_{i}\right) \times P\left(b_{i} \mid \tau\right) P(\tau)
\end{aligned}
$$

The relationship between the observed deaths from breast cancer and detection modes (the participant, the non-participant, and the uninvited groups) is programmed in the right side of the Fig. 2, which is linked to the left side of attendance rate based on the observed data on the participant and the non-participant groups to estimate adjRR as mentioned above.

As mentioned in the methodological section, we used two types of prior distribution. The use of different prior distributions is specified in Table 3. The non-informative priors for alpha and beta follows a normal distribution denoted as $N\left(0,10^{6}\right)$. The second type is informative priors for $\alpha$ and $\beta \mathrm{s}$, following normal distribution with parameters
Table 4 Number of participants, person-years, and breast cancer death of four studies

\begin{tabular}{llll}
\hline Country/region [references] & Attenders & Non-attenders & Control \\
\hline Person-year & & & \\
Dalarna [9] & 171,567 & 19,063 & 90,523 \\
Gothenburg [10] & 39,651 & 9,913 & 78,369 \\
Finnish [3] & 349,679 & 51,125 & 299,228 \\
Pirkanmaa (current) & 233,991 & 14,966 & N/A \\
Subjects & & & \\
Dalarna [9] & 10,555 & 1,173 & 5,557 \\
Gothenburg [10] & 7,941 & 1,985 & 15,744 \\
Finnish [3] & 76,389 & 13,504 & 68,862 \\
Pirkanmaa (current) & 84,812 & 10,245 & N/A \\
Breast cancer death & & & \\
Dalarna [9] & 29 & 8 & 38 \\
Gothenburg [10] & 24 & 14 & 66 \\
Finnish [3] & 49 & 15 & 63 \\
Pirkanmaa & 29 & 7 & N/A \\
\hline
\end{tabular}

(mean and variance) borrowing prior information either from previous studies by Hakama et al. or from other relevant studies from literatures. For prior information using the Hakama study, the posterior distribution that combines the prior information with the likelihood based on data from the Pirkanmaa was computed and the corresponding adjusted RR was also calculated. The estimated results with non-informative and informative priors borrowing from the Hakama study only are shown in Table 3. Table 3 also shows the incorporation of prior distribution in a sequential way with chronological order for which the posterior distribution of earlier study was treated as the prior distribution for the next study. Namely, to obtain the abovementioned adjRR in a service screening program in Pirkanmaa, Finland, we retrieved data from three other breast cancer screening programs from Sweden. Data are listed in a chronological order in Table 4. The service screening program was conducted in Pirkanmaa since 1988. Three studies were Dalarna County [9], Gothenburg [10], and Hakama's [3] conducted since 1977, 1982, and 1987, respectively. Therefore, we first estimated parameters starting from Dalarna County in Sweden. The estimated parameters of posterior distribution from Dalarna County in Sweden were used as the informative prior for the second study, the Gothenburg trial. The same procedure was applied to Hakama's, and then to Pirkanmma. These estimated results are shown in Table 3. For parameter estimation, we used the Gibbs sampler to implement the MCMC method and obtain the marginal posterior distributions for parameters of interests following Spiegelhalter et al. [13] method. 


\section{References}

1. Sarkeala T, Anttila A, Forsman H, Luostarinen T, Saarenmaa I, Hakama M (2004) Process indicators from ten centres in the Finnish breast cancer screening programme from 1991 to 2000. Eur J Cancer 40:2116-2125. doi:10.1016/j.ejca.2004.06.017

2. Sarkeala T, Anttila A, Saarenmaa I, Hakama M (2005) Validity of process indicators of screening for breast cancer to predict mortality reduction. J Med Screen 12:33-37

3. Hakama M, Pukkala E, Heikkilä M, Kallio M (1997) Effectiveness of the public health policy for breast cancer screening in Finland: population based cohort study. Br Med J 314:864-867

4. Seppänen J, Heinävaara S, Anttila A, Sarkeala T, Virkkunen H, Hakulinen T (2006) Effects of different phases of an invitational screening program on breast cancer incidence. Int $\mathrm{J}$ Cancer 119:920-924. doi:10.1002/ijc.21870

5. Dean PB, Pamilo M (1999) Screening mammography in Finland-1.5 million examinations with 97 percent specificity. Acta Oncol Suppl 13:47-54

6. Parvinen I, Helenius H, Pylkkänen L, Anttila A, Immonen-Räihä P, Kauhava L, Räsänen O, Klemi PJ (2006) Service screening mammography reduces breast cancer mortality among elderly women in Turku. J Med Screen 13:34-40

7. Aro AR, Koning HJ, Absetz P, Schreck M (2001) Two distinct groups of non-attenders in an organized mammography screening program. Breast Cancer Res Treat 70:145-153
8. Cook TD, Campbell DT (1979) Quasi-experiments: nonequivalent control group designs. In: Quasi-experimentation: design \& analysis issues for field settings. Houghton Mifflin Co, Boston, pp 98-103

9. Tabár L, Vitak B, Chen HH, Duffy SW, Yen MF, Chiang CF, Krusemo UB, Tot T, Smith RA (2000) The Swedish two-county trial twenty years later. Updated mortality results and new insights from long-term follow-up. Radiol Clin North Am 38:625-651

10. Bjurstam N, Björneld L, Warwick J, Sala E, Duffy SW, Nyström L (2003) The Gothenburg breast screening trial. Cancer 97:23872396

11. Tabar L, Yen MF, Vitak B, Chen HH, Smith RA, Duffy SW (2003) Mammography service screening and mortality in breast cancer patients: 20-year follow-up before and after introduction of screening. Lancet 361:1405-1410

12. Duffy SW, Cuzick J, Tabar L, Vitak B, Chen THH, Yen MF, Smith RA (2002) Correcting for non-compliance bias in casecontrol studies to evaluate cancer screening programmes. Appl Statist 51:235-243

13. Spiegelhalter DJ, Thomas A, Best NG, Gilks WR (1996) BUGS 0.5 , Bayesian Inference Using Gibbs Sampling. Manual (Version ii). MRC Biostatistics Unit, Cambridge 